\title{
Phase-field simulation and design of a ferroelectric nano-generator
}

\author{
M. Krauß ${ }^{a}$, I. Münch ${ }^{a}$, C. M. Landis ${ }^{b}$ and W. Wagner ${ }^{a}$ \\ ${ }^{a}$ Institute for Structural Analysis, Karlsruhe Institute of Technology (KIT), Kaiserstr. 12, \\ 76133 Karlsruhe, Germany; \\ ${ }^{b}$ Aerospace Engineering and Engineering Mechanics, University of Texas at Austin, Austin, \\ United States
}

\begin{abstract}
We study the behavior of ferroelectric material $\left(\mathrm{BaTiO}_{3}\right)$ for the design of a nano-generator to convert mechanical into electrical energy. The investigations consider an electro-mechanical phase-field model with polarization as state variable. This widely accepted model has its origins in the work of ${ }^{1-3}$ and is fully developed by Landis and coworkers. ${ }^{4,5}$ We use a finite element model to simulate tetragonal regions of ferroelectric material sputtered on substrate. Different geometries as well as various mechanical and electrical boundary conditions are considered. The model parameters are normalized to achieve better computational conditions within the stiffness matrix. The major objective of this contribution is the fundamental understanding of domain switching caused by a cyclic electrical field. The corresponding hysteresis loops of the overall polarization cannot be achieved by using a two-dimensional model because the domain topologies evolve in three dimensions. The three-dimensional nature of the domain structure evolution is even true for flat regions or thin films. ${ }^{6}$ We show some examples of three-dimensional domain topologies, which are able to break energetically unfavorable symmetries. Finally, the computational model of a tetragonal nano-generator with dimensions $10 \times 60 \times 10 \mathrm{~nm}$ is presented. The specific ratio of height to width and the mounting on substrate is essential for its performance and principle of energy harvesting. We discuss the challenges and scopes of such a system.
\end{abstract}

Keywords: Phase-field modeling, ferroelectricity, nano-generator, epitaxial strain, finite element method, energy-harvesting.

\section{INTRODUCTION}

Scientific research within the last two decades has given great interest to ferroelectric materials ${ }^{7-9}$ as a subset of smart materials. Below the Curie temperature ferroelectrics consist of domains of aligned electric polarization following electrical and mechanical conditions. The coupling between electrical and mechachical fields enables the conversion of mechanical into electrical energy. The continual success in miniaturizing electronic devices motivates our work for energy harvesting on the nano-scale. We simulate structured ultrathin layers of ferroelectric material epitaxially sputtered onto a substrate. Experimental and theoretical results in ${ }^{10,11}$ show that ferroelectricity exists down to a thickness of $3 \mathrm{~nm}$. Furthermore it is well known that the material properties of epitaxially deposited films are affected by the crystal structure of the underlying substrate. Thin films conform to the lattice parameters of the substrate which cause misfit strains at the interface between the ferroelectric film and substrate. Experimental work, see e.g. ${ }^{12-15}$ as well as theoretical work, see e.g. ${ }^{16-22}$ discuss this effect as a material design parameter. Epitaxial strain even controls the transition from paraelectric to ferroelectric behavior. This feature is frequently investigated by using phase-field modeling which is in agreement with experimental data. ${ }^{23,24}$ To determine intrinsic domain evolution mechanisms phase-field modeling is an effective method since all necessary electrical and mechanical boundary conditions can be incorporated.

Further author information: (Send correspondence to M. Krauß)

M.K.: E-mail: matthias.krauss@kit.edu, Telephone: +49 721 608-42285

I.M.: E-mail: ingo.muench@kit.edu, Telephone: +49 721 608-42289

C.M.L.: E-mail: landis@mail.utexas.edu, Telephone: +01 512-471-4273

W.W.: E-mail: w.wagner@kit.edu, Telephone: +49 721 608-42280

Behavior and Mechanics of Multifunctional Materials and Composites 2011,

Edited by Zoubeida Ounaies, Stefan S. Seelecke, Proc. of SPIE Vol. 7978, 797821

(c) 2011 SPIE · CCC code: 0277-786X/11/\$18 $\cdot$ doi: $10.1117 / 12.880493$

Proc. of SPIE Vol. $7978797821-1$ 
The article is organized as follows. Section 2 presents the governing equations of the phase-field model and discuss its normalization. In Section 3 computational results on nano-scaled cubes are presented. The examples show the effect of altered geometries and interfacial strain. Additionally, the need for three-dimensional modeling is illustrated. Next, a nano-scaled energy converter is discussed in Section 4. Finally, Section 5 gives conclusions and an outlook on further research.

\section{FOUNDATIONS OF THE MODEL}

$\mathrm{ABO}_{3}$-type perovskites undergoing the phase transition from cubic into tetragonal crystal structure have six variants: polarization along or opposite to the [100], [010], and [001] directions of the unit cell. Polarization is accompanied with lattice distortion (electrostriction) resulting in spontaneous strain $\varepsilon_{0}=\frac{c-a}{a}>0$, see Fig. 1. Any insulator is electrostrictive but in ferroelectrics the quadratic coupling between polarization and strain is exceptionally strong. Thus, the parameters $b_{i j k l}$ in Eq. 10 are relatively large. Both, the material
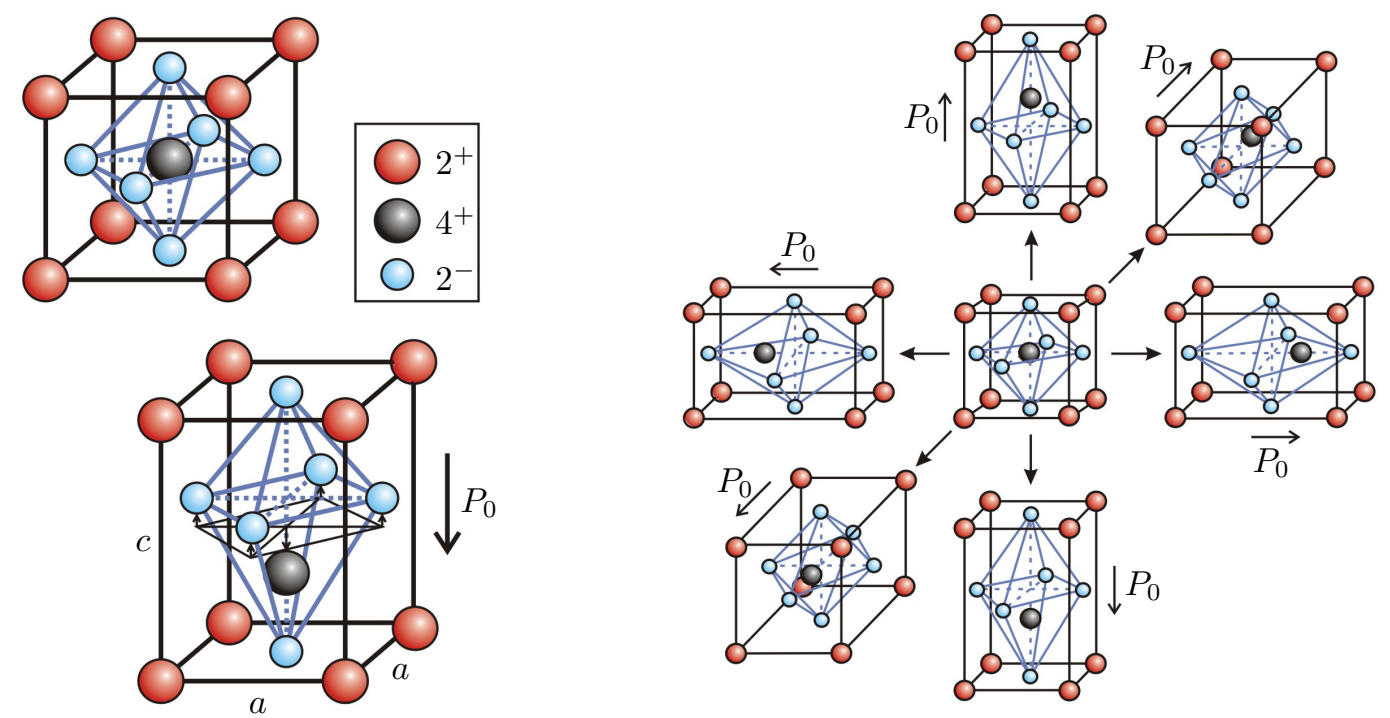

Figure 1. A schematic of the behavior of $\mathrm{ABO}_{3}$-type ferroelectrics undergoing a phase transition below the Curie temperature. The letters A and B indicate metal ions with $2^{+}$resp. $4^{+}$valence electrons. In the ferroelectric phase the unit cell becomes tetragonal with asymmetrically distributed electric charges causing spontaneous polarization $P_{0}$. The six variants of the ferroelectric phase are characterized by the polarization vectors.

polarization $^{5,16,25}$ or the spontaneous polarization, see e.g. ${ }^{23,26}$ are possible primary thermodynamic order parameters leading to different phase-field models but similar results. Considering the material polarization $\mathbf{P}$ the electric displacement reads

$$
\mathbf{D}:=\mathbf{P}+\kappa_{\mathbf{0}} \mathbf{E}
$$

with the electric field $\mathbf{E}$ and the permittivity of the free space $\kappa_{0}=8.854 \cdot 10^{-12} \mathrm{Vm} / \mathrm{C}$. Within the ferroelectric body $\mathcal{B}$ the electrostatic field equations are fulfilled through

$$
\begin{aligned}
\mathbf{E}:=-\operatorname{Grad}[\varphi] & \text { in } \mathcal{B} \\
\operatorname{Div}[\mathbf{D}]=q & \text { in } \mathcal{B}
\end{aligned}
$$

where $\varphi$ is the electric potential and $q$ is the volume charge density. On the electroded surface $\partial \mathcal{B}_{e}$ and on the insulated surface $\partial \mathcal{B}_{i}$ it holds

$$
\mathbf{D} \cdot \mathbf{n}=\omega \text { on } \partial B
$$

The unit vector $\mathbf{n}$ is normal to the surface, and $\omega$ is the surface charge density. The mechanical part of the model assumes linear kinematics. Thus, the mechanical strain is defined by

$$
\varepsilon:=\frac{1}{2}\left(\operatorname{Grad}[\mathbf{u}]+\operatorname{Grad}^{\mathrm{T}}[\mathbf{u}]\right)=\operatorname{Sym}\left[\frac{\partial \mathbf{u}}{\partial \mathbf{x}}\right],
$$


and the standard equilibrium equations appear as

$$
\begin{aligned}
\operatorname{Div}[\boldsymbol{\sigma}]+\mathbf{b}=\mathbf{0} & \text { in } \mathcal{B} \\
\boldsymbol{\sigma}=\boldsymbol{\sigma}^{\mathrm{T}} & \text { in } \mathcal{B} \\
\boldsymbol{\sigma} \cdot \mathbf{n}=\overline{\mathbf{t}} & \text { on } \partial \mathcal{B}_{t} \\
\mathbf{u}=\overline{\mathbf{u}} & \text { on } \mathcal{B}_{u} .
\end{aligned}
$$

Herein, $\mathbf{u}$ is the displacement vector, $\boldsymbol{\sigma}$ the Cauchy stress tensor, $\mathbf{b}$ are body forces per unit volume, and $\mathbf{t}$ are tractions applied on the surface. Following the Landau-Devonshire theory ${ }^{27}$ the Helmholtz free energy density for ferroelectrics having centrosymmetric parent phase reads in indicial notation ${ }^{5,16}$

$$
\begin{aligned}
\psi & =\frac{1}{2} a_{i j k l} P_{i, j} P_{k, l}+\frac{1}{2} \bar{a}_{i j} P_{i} P_{j}+\frac{1}{4} \overline{\bar{a}}_{i j k l} P_{i} P_{j} P_{k} P_{l}+\frac{1}{6} \overline{\bar{a}}_{i j k l m n} P_{i} P_{j} P_{k} P_{l} P_{m} P_{n} \\
& +\frac{1}{8} \overline{\overline{\bar{a}}}_{i j k l m n r s} P_{i} P_{j} P_{k} P_{l} P_{m} P_{n} P_{r} P_{s}-b_{i j k l} \varepsilon_{i j} P_{k} P_{l}+\frac{1}{2} c_{i j k l} \varepsilon_{i j} \varepsilon_{k l} \\
& +f_{i j k l m n} \varepsilon_{i j} \varepsilon_{k l} P_{m} P_{n}+g_{i j k l m n} \varepsilon_{i j} P_{k} P_{l} P_{m} P_{n}+\frac{1}{2 \kappa_{0}}\left(D_{i}-P_{i}\right)^{2} .
\end{aligned}
$$

The coefficients $a_{i j k l}=a_{0}$ for $i j=k l$ give an isotropic energy penalty for spatially inhomogeneous polarization. The thickness of the domain walls is a characteristic material length scale and is connected to $a_{0}$. The dielectric stiffness $\bar{a}_{i j}$ together with higher order terms indicated by multiple bars provides an energy well structure corresponding to the six stable domain states of the polar tetragonal crystal system. The piezoelectric and elastic properties of monodomain single crystal $\mathrm{BaTiO}_{3}$ near the spontaneous polarization $P_{0}=0.26 \mathrm{C} / \mathrm{m}^{2}$ and the spontaneous strain $\varepsilon_{0}=0.82 \%$ are given through the tensors $b_{i j k l}, c_{i j k l}, f_{i j k l m n}$ and $g_{i j k l m n}$. The permittivity $\kappa_{0}$ accounts for the stored electric energy of the free space occupied by the material. For further details on the specific form of the free energy with characteristic material parameters for $\mathrm{BaTiO}_{3}$ we refer to the work of Landis and coworkers. ${ }^{16}$

The polarization is the state variable of the phase field model and evolves according to the Ginzburg-Landau equations

$$
\left(\frac{\partial \psi}{\partial P_{i, j}}\right)_{, j}-\frac{\partial \psi}{\partial P_{i}}=\beta \dot{P}_{i} \quad, \quad \beta \geq 0
$$

where $\beta$ represents the polarization viscosity. Eqs. 1-11 are implemented using a finite element formulation. The seven nodal degrees of freedom of the finite elements are the mechanical displacements, the material polarization and the electric potential $\phi$. The electric field enters the formulation by the electric enthalpy $h$, which is obtained from the Helmholtz free energy density $\psi$ via the Legendre transformation $h=\psi-E_{i} D_{i}$. Strain, polarization, polarization gradient and electric field are interpolated within the finite element using isoparametric trilinear shape functions. The Ginzburg-Landau equation as well as mechanical and electrical equilibrium equations can be expressed in a virtual work statement

$$
\int_{\mathrm{V}}\left(\beta \dot{P}_{i} \delta P_{i}+\sigma_{i j} \delta \varepsilon_{i j}+D_{i} \delta E_{i}+\eta_{i} \delta P_{i}+\xi_{i j} \delta P_{i, j}\right) d \mathrm{~V}=0
$$

with $\sigma_{i j}=\partial h / \partial \varepsilon_{i j}=\partial \psi / \partial \varepsilon_{i j}, D_{i}=-\partial h / \partial E_{i}, \eta_{i}=\partial h / \partial P_{i}=\partial \psi / \partial P_{i}$ and $\xi_{i j}=\partial h / \partial P_{i, j}=\partial \psi / \partial P_{i, j}$. Eq. 12 is solved in the finite element formulation using a standard Newton-Raphson scheme. Additionally, a first-order accurate backward Euler scheme is used to discretize the polarization rate within each time step.

\subsection{Normalizations}

Here we give some explanation to the normalization of the phase-field model we use. The aim is to transform the Helmholtz free energy density, the length scale, and the mechanical strain such that they appear as dimensionless quantities. The normalization factors are physical material parameters namely the coercive field for homogeneous $180^{\circ}$ switching $E_{c}$, the spontaneous polarization $P_{0}$, and the spontaneous strain $\varepsilon_{0}$. Additionally, the length scale $l_{0}=1 \mathrm{~nm}$ is employed and connected to the thickness of a $180^{\circ}$ domain wall, see Sec. 2.2. The normalization 
factors are directly related to the electric field $\mathbf{E}$, the material polarization $\mathbf{P}$, the geometry of the structure parameterized by coordinates $\mathbf{x}$, and the mechanical strain $\varepsilon$ as follows

$$
\begin{array}{llll}
\mathbf{E} & \mapsto \quad \overline{\mathbf{E}}:=\frac{\mathbf{E}}{E_{c}} & \mathbf{x} & \mapsto \quad \overline{\mathbf{x}}:=\frac{\mathbf{x}}{l_{0}} \\
\mathbf{P} & \mapsto \quad \overline{\mathbf{P}}:=\frac{\mathbf{P}}{P_{0}} & \varepsilon & \mapsto \bar{\varepsilon}:=\frac{\varepsilon}{\varepsilon_{0}} .
\end{array}
$$

Defining

$$
\overline{\kappa_{0}}:=\frac{\kappa_{0} E_{c}}{P_{0}}
$$

the normalized electric displacement reads

$$
\mathbf{D} \mapsto \overline{\mathbf{D}}:=\overline{\mathbf{P}}+\overline{\kappa_{0}} \overline{\mathbf{E}}=\frac{\mathbf{P}}{P_{0}}+\frac{\kappa_{0} E_{c}}{P_{0}} \frac{\mathbf{E}}{E_{c}}=\frac{\left(\mathbf{P}+\kappa_{0} \mathbf{E}\right)}{P_{0}}=\frac{\mathbf{D}}{P_{0}} .
$$

Considering the Helmholtz free energy density to be normalized via electric parameters results in

$$
\psi \mapsto \quad \mapsto \bar{\psi}:=\frac{\psi}{E_{c} P_{0}} .
$$

The electric enthalpy transforms via

$$
h:=\psi-\mathbf{E} \cdot \mathbf{D} \quad \mapsto \quad \bar{h}:=\frac{h}{E_{c} P_{0}}=\frac{\psi-\mathbf{E} \cdot \mathbf{D}}{E_{c} P_{0}}=\frac{\psi}{E_{c} P_{0}}-\frac{\mathbf{E}}{E_{c}} \cdot \frac{\mathbf{D}}{P_{0}}=\bar{\psi}-\overline{\mathbf{E}} \cdot \overline{\mathbf{D}} .
$$

The normalization of length scale and mechanical strain yields

$$
\overline{\boldsymbol{\varepsilon}}:=\frac{\boldsymbol{\varepsilon}}{\varepsilon_{0}}=\operatorname{Sym}\left[\frac{1}{\varepsilon_{0}} \frac{\partial \mathbf{u}}{\partial \mathbf{x}}\right]=\operatorname{Sym}\left[\frac{\partial}{\partial \overline{\mathbf{x}}}\left(\frac{\mathbf{u}}{l_{0} \varepsilon_{0}}\right)\right]=\operatorname{Sym}\left[\frac{\partial \overline{\mathbf{u}}}{\partial \overline{\mathbf{x}}}\right]
$$

leading to the definition of the normalized displacement as

$$
\mathbf{u} \mapsto \quad \overline{\mathbf{u}}:=\frac{\mathbf{u}}{\varepsilon_{0} l_{0}} .
$$

A closer look at the electric field

$$
\overline{\mathbf{E}}:=\frac{\mathbf{E}}{E_{c}}=\frac{1}{E_{c}} \frac{\partial \phi}{\partial \mathbf{x}}=\frac{\partial}{\partial \overline{\mathbf{x}}}\left(\frac{\phi}{l_{0} E_{c}}\right)=\frac{\partial \bar{\phi}}{\partial \overline{\mathbf{x}}}
$$

gives the normalization for the electric potential

$$
\phi \quad \mapsto \quad \bar{\phi}:=\frac{\phi}{E_{c} l_{0}} .
$$

Details on the transformation for the coefficients in Eq. 10 are given in the Appendix A. The normalizations have great benefit for the finite element model. The mechanical and electrical parts within the stiffness matrix become similar magnitudes. In our simulations we observe the minimum and maximum values of the diagonal components within the stiffness matrix which produce a ratio of about $\approx 10^{4}$. This ratio depends on the specific example, the finite element mesh and the material parameters.

\subsection{Association of domain wall thickness with the length scale}

The normalized phase-field model allows for a direct connection of the internal length scale $l_{0}$ to the thickness of a domain wall. Without limiting the generality we sketch the assumptions for such a relation in Fig. 2 for a 
$180^{\circ}$ domain wall. According to Eq. 10 the polarization gradient leads to a Helmholtz free energy density given by

$$
\psi_{\nabla \mathrm{P}}=\frac{1}{2} a_{0}\left(\frac{\partial \mathbf{P}}{\partial \mathbf{x}}\right)^{2}
$$

The actual variation of $\mathrm{P}$ within the domain wall is sketched as nonlinear curve in Fig. 2. However, considering the linearization of the curve in the center of the wall at $\bar{x}=0$ results in a tangent $\frac{\partial \bar{P}}{\partial \bar{x}}=1$ becoming $\bar{P}=1$ at $\bar{x}=1$. With this assumption the normalized Helmholtz free energy density reads

$$
\bar{\psi}_{\nabla \mathrm{P}}:=\frac{\psi_{\nabla \mathrm{P}}}{P_{0} E_{c}}=\frac{1}{2} a_{0} \frac{P_{0}}{E_{c}}\left(\frac{\partial\left(\mathbf{P} / P_{0}\right)}{\partial \mathbf{x}}\right)^{2}=\frac{1}{2} a_{0} \frac{P_{0}}{E_{c}}\left(\frac{\partial \overline{\mathbf{P}}}{\partial \overline{\mathbf{x}} l_{0}}\right)^{2}=\frac{1}{2} a_{0} \frac{P_{0}}{E_{c} l_{0}{ }^{2}}(1)^{2} .
$$

Enforcing the energy to fulfill $\int_{-1}^{1} \bar{\psi}_{\nabla \mathrm{P}} \mathrm{d} \bar{x}=1$ yields the material parameter

$$
a_{0}=\frac{E_{c} l_{0}^{2}}{P_{0}}
$$

In other words, considering Eq. 24 in the model leads to a $180^{\circ}$ domain wall thickness of $2 l_{0}$.

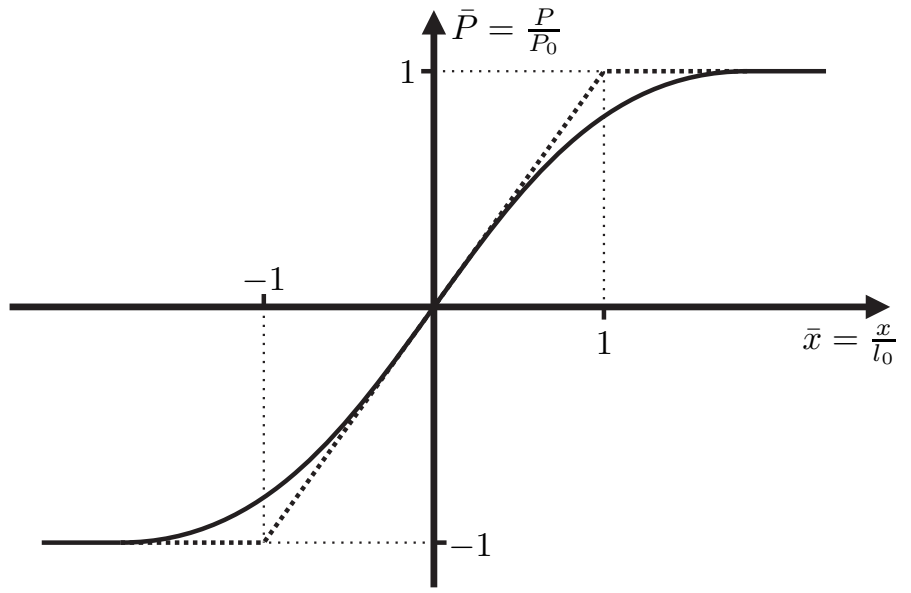

Figure 2. Phase transition from $\bar{P}=-1$ to $\bar{P}=1$ within a $180^{\circ}$ domain wall.

\section{ELECTRICAL HYSTERESIS OF FERROELECTRIC CUBES ON SUBSTRATE}

In this section computational investigations are performed on ferroelectric nano-cubes unidirectionally strained at the interface by an underlying substrate. We focus on the influence of that strain onto the polarization behavior of the cube due to an applied electric field. However, the question arises, how can such a specific unidirectional strain can be achieved. Our idea is to use $\mathrm{MgAl}_{2} \mathrm{O}_{3}$ as substrate offering a nearly perfect lattice match to $\mathrm{BaTiO}_{3}$. Practically, the non-conductive $\mathrm{MgAl}_{2} \mathrm{O}_{3}$ substrate would be covered with a thin platinum layer. Experiments have shown that thin films of $\mathrm{Pt}$ can grow epitaxially on $\mathrm{MgAl}_{2} \mathrm{O}_{3}$ retaining the lattice parameters of the substrate. At the interface with the ferroelectric film the epitaxial strain (which would be biaxial) vanishes and the sputtering process is more stable as compared to the case with lattice misfit. The unidirectional interfacial strain $\varepsilon_{i f}$ can be enforced by adhering the substrate onto a base layer, which is initially prestressed. After the drying time for the adhesive the prestress onto the base is released and a specific deformation results for the substrate. This deformation can be designed such that an unidirectional strain at the interface to the ferroelectric appears. For our investigations we use $\varepsilon_{i f}$ as design parameter and relate it to the materials spontaneous strain value $\varepsilon_{0}$. As sketched in Fig. 3 the cubes are electroded on the top and bottom surfaces. Their dimension is 


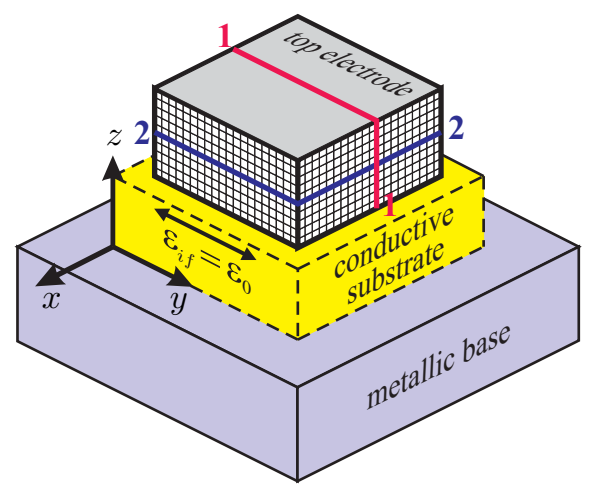

Figure 3. Configuration of the simulation model showing the finite element mesh and cuts along the cross sections 1-1 and $2-2$.

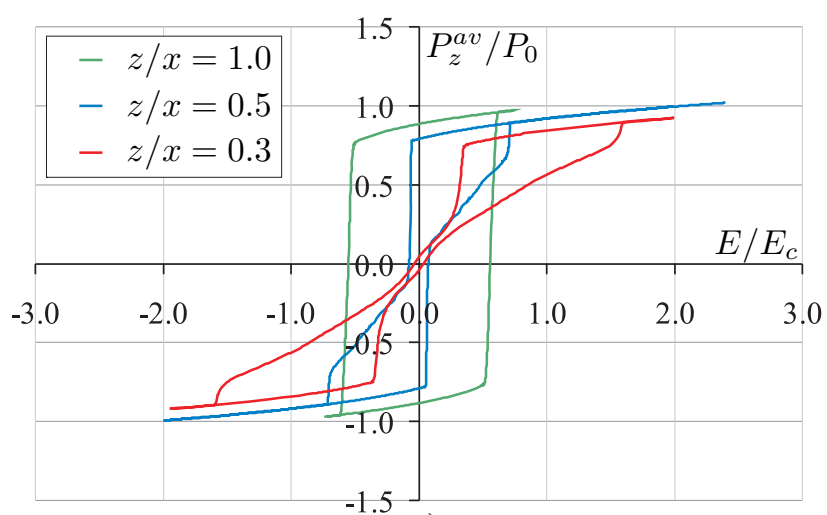

a)

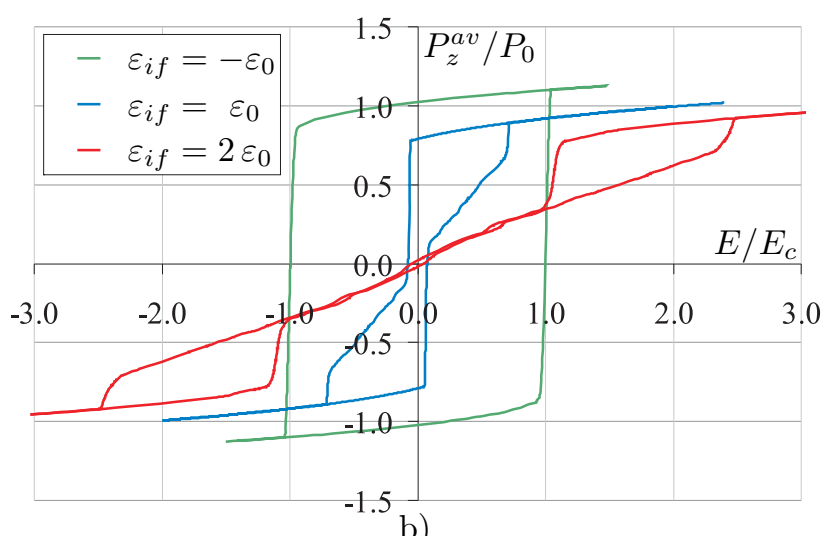

b)

Figure 4. a) Electric hysteresis-loops of ferroelectric nano-cubes underlying constant epitaxial strain $\varepsilon_{i f}=\varepsilon_{0}$ but different aspect-ratios $z / x$. b) Electric hysteresis-loops of a ferroelectric nano-cube with dimensions $20 \times 20$ x $10 \mathrm{~nm}$ (aspect-rate $z / x=0.5)$ underlying different interfacial strains $\varepsilon_{i f}$.

20 by $20 \mathrm{~nm}$ in the $x$ - $y$-plane and the height in z-direction varies. Thus, the aspect ratio of height and width is another design parameter in this study.

For the finite element simulation the nano-cubes are discretized with cubic elements of $1 \mathrm{~nm}$ edge length. Compare that the unit cell of $\mathrm{BaTiO}_{3}$ is $0.4 \mathrm{~nm}$ and the thickness of a $90^{\circ}$ domain wall is discussed in literature between 2 to $25 \mathrm{~nm} .{ }^{28}$ Our phase field model considers a $90^{\circ}$ domain wall about $4 \mathrm{~nm}$ thickness and consequently we use enough elements to avoid mesh pinning as numerical pre-studies have shown. The diagrams in Fig. 4 and Fig. 5 consider the averaged polarization in z-direction according to

$$
P_{z}^{a v}=\frac{\int_{\mathcal{B}} P_{z} \mathrm{~d} V}{\int_{\mathcal{B}} \mathrm{d} V} .
$$

A cyclic electric field is applied between the top and bottom electrode. The normalized hysteresis loop for $\mathrm{P}_{z}^{a v}$ as a function of the normalized electrical field $E / E_{c}$ is shown in Fig. 4. The calculations demonstrate that the intrinsic ferroelectric material behavior represented by $\mathrm{P}_{z}^{a v}$ can be designed and controlled by extrinsic constraints. Interfacial strain as well as the geometry of the ferroelectric are effective parameters to design the material response. The interfacial strain even controls the transition from paraelectric to ferroelectric behavior. Compare Fig. $4 \mathrm{~b}$ ) where the interfacial strain $\varepsilon_{i f}=2 \varepsilon_{0}$ enforces the material to become paraelectric in z-direction below the coercive field $\mathrm{E}_{c}$.

Next, we demonstrate the deficiency of a $2 \mathrm{D}$ calculation by comparing its results to the $3 \mathrm{D}$ simulation. Focus is given to a nano-cube with dimensions 20 by $20 \mathrm{~nm}$ and $10 \mathrm{~nm}$ height and unidirectional interfacial strain 


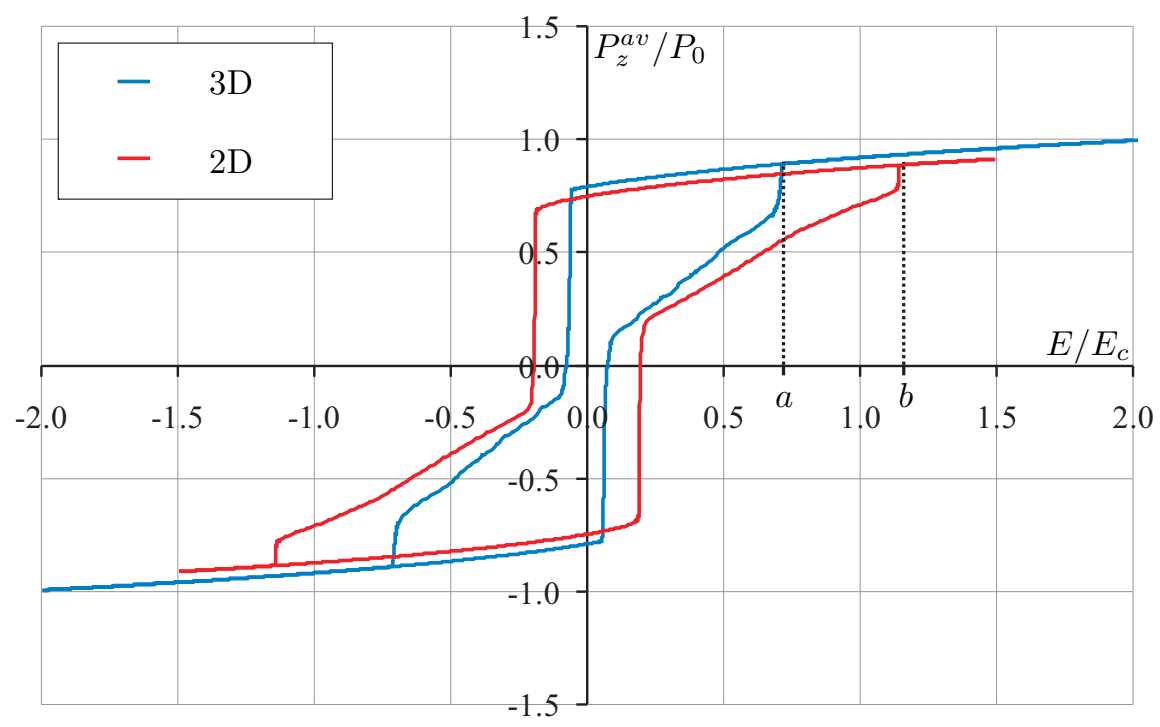

Figure 5. Comparison of the hysteresis loops in two and three dimensional simulations for the $20 \mathrm{x} 20 \mathrm{x} 10 \mathrm{~nm}$ cube under a cyclic electric field. The characteristic values $a=E_{c z}^{3 D} / E_{c}$ and $b=E_{c z}^{2 D} / E_{c}$ are indicated by dotted lines.
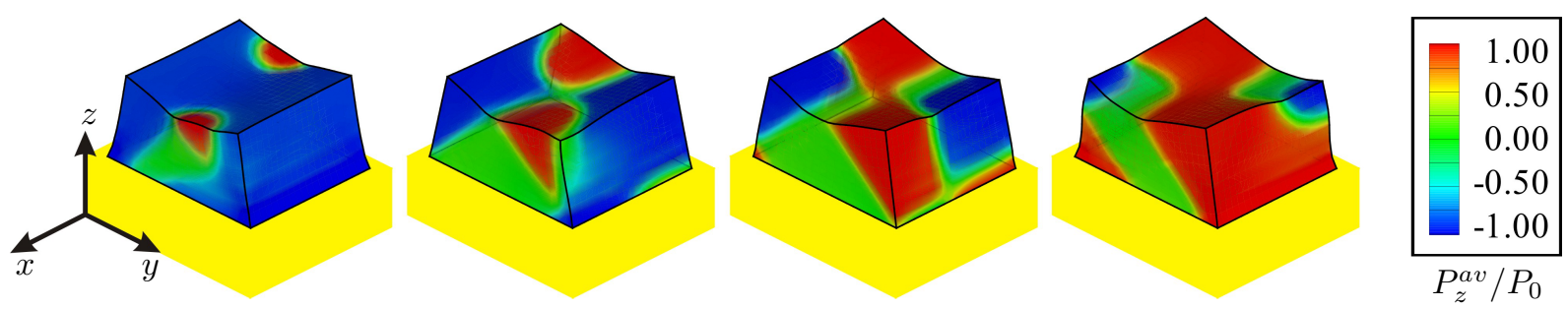

Figure 6. Domain switching sequence of a nano-cube with the height of $10 \mathrm{~nm}$ under a cyclic electric field. Polarization is depicted by colors and the deformation is exaggerated.

$\varepsilon_{i f}=\varepsilon_{0}$ within a cyclic electric field. In the two-dimensional case the cube is modeled in the direction of the interfacial strain and assumes plane strain $\varepsilon_{i f}=0$ in the third direction. The 3D cube is discretized with 20 by 20 by 10 elements, whereas the $2 \mathrm{D}$ simulation uses 200 by 100 elements. The resulting hysteresis loops can be seen in Fig. 5. The graphs show significant differences, e.g. the normalized coercive field value $E_{c z}^{2 D} / E_{c}$ and $E_{c z}^{3 D} / E_{c}$. These discrepancies can be illuminated by comparing the domain mechanisms within the cubes. The pictures in Fig. 6 show domain states due to a polarization shift from negative to positive z-direction caused by an increasing electric field in positive z-direction. The domain topology during the switching process is quite three-dimensional. Domains are asymmetrically arranged at the top of the structure and evolve mainly along the diagonal axis. This feature is a-priori excluded in a 2D model.

In Fig. 7 the cross sections along the 1-1-plane for the 3D and the 2D simulation can be compared. The domain state $i v$ ) illustrates the shortcomings in case of 2D investigations, since energetically unfavorable domain topologies cannot be compensated via interactions in lateral direction. Fig. 8 shows the rotational behaviour of the domain shifting process. Such behavior can not be described with 2D models since the domains evolve three-dimensionally. 
a)

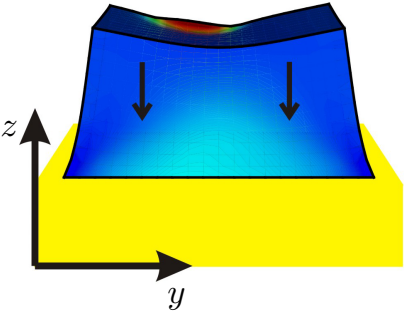

i)

b)

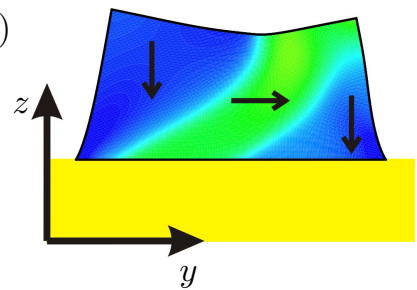

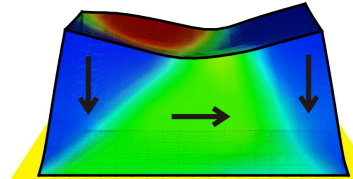

ii)

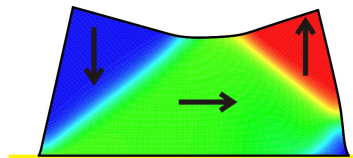

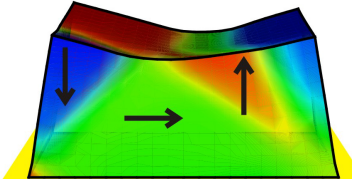

iii)

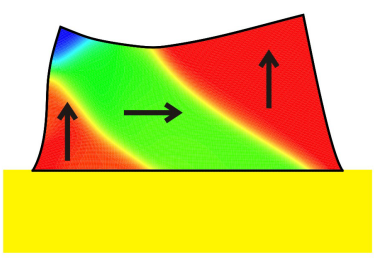

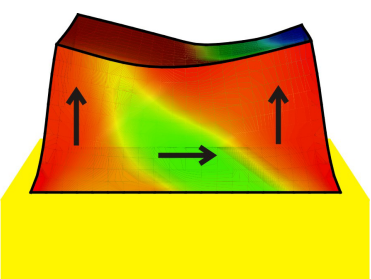

$i v)$

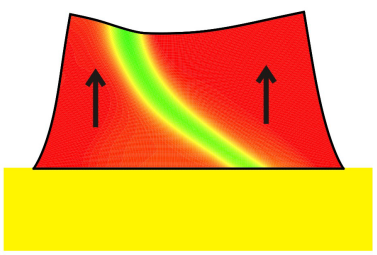

Figure 7. a) Cross sections along the 1-1-plane due to a vertical cut of the three-dimensional nano-cube. b) Results of the two-dimensional simulation. Polarization is depicted by colors and arrows and the deformation is exaggerated.

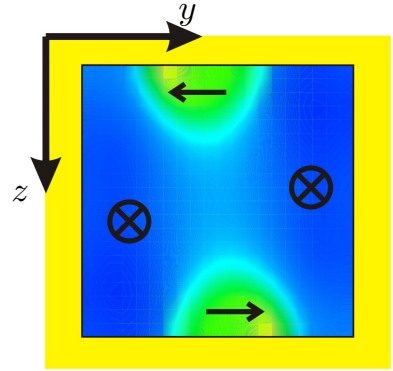

a)

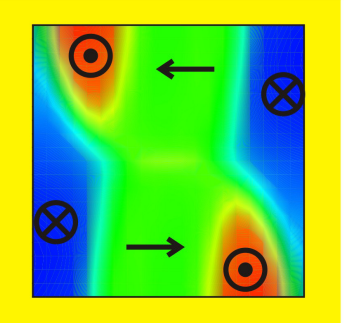

b)

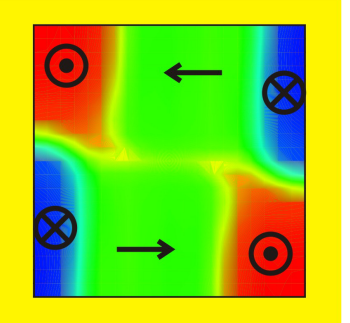

c)

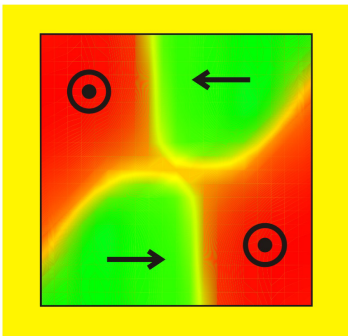

d)

Figure 8. a) - d) Transverse cross sections along the 2-2-plane due to a horizontal cut and formation of the domain mechanisms. Polarization is depicted by colors and arrows.

\section{ELECTRO-MECHANICAL HYSTERESIS OF AN ENERGY CONVERTER}

This numerical example simulates a structured $\mathrm{BaTiO}_{3}$ thin film of $10 \mathrm{~nm}$ thickness deposited on an electrically conductive substrate with perovskite structure. The assumed biaxial epitaxial strain of $\varepsilon_{\text {if }}=0.5 \varepsilon_{0}=0.41 \%$ is provoked by distinct lattice parameters of the substrate and ferroelectric. It causes biaxial tensile stresses in the ferroelectric material at the interface with the substrate. We consider a ferroelectric single crystal with square dimensions of $10 \mathrm{~nm}$ in the lateral directions and $60 \mathrm{~nm}$ in the longitudinal direction, see Fig. 9. The top electrode may be designed as a lithographically patterned platinum layer that covers $75 \%$ of the surface, as depicted in Fig. 9. The finite element mesh for the simulation uses 16 elements in lateral direction, 96 elements in longitudinal direction and 16 elements in thickness direction. Thus, each element is cubic with an edge length of $0.625 \mathrm{~nm}$. The simulation starts with an initial poling process by employing a potential difference between the top and bottom electrode, compare Fig. 9. The result is an initial polarization arranged in three domains, similar to the domain topology in Fig. $10 \mathrm{c}$ ). After the poling process the domain alignment evolves into a stable topology as shown in Figure 10 a).

During the straining process the conductive substrate is strained by a homogeneous mechanical deformation. A simple bending mechanism can be used to cause such a mechanical deformation of the substrate. We simulate a first hysteresis loop $\varepsilon_{i f} \in\left[0,0.5 \varepsilon_{o}\right]$ and a second loop $\varepsilon_{i f} \in\left[-0.3 \varepsilon_{o}, 0.5 \varepsilon_{o}\right]$. In the first loop the cyclic deformation process starts with the intrinsic strain of $\varepsilon_{i f}=0.5 \varepsilon_{0}=0.41 \%$ and reaches the value of $\varepsilon_{i f}=0$. Compressing the substrate periodically leads to a fully reversible domain shifting process; compare Fig. 10 a) - c). 


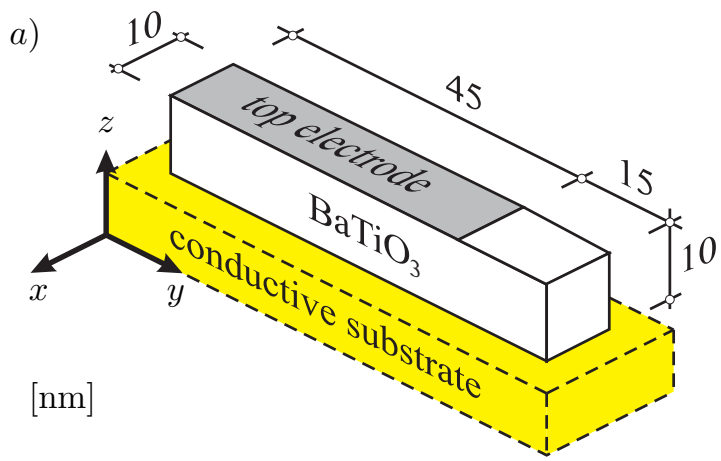

b)
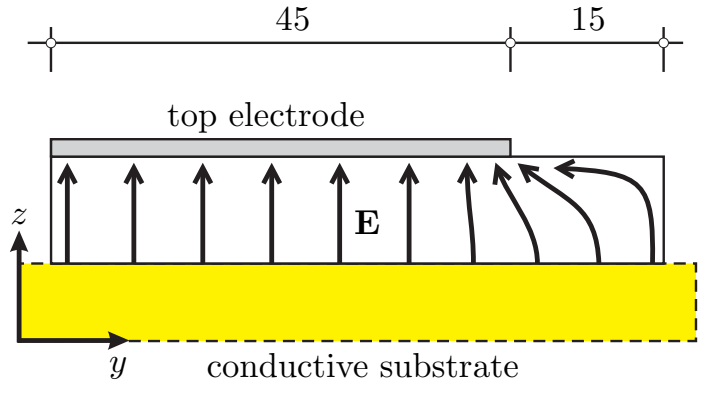

Figure 9. Design and dimensions of the nano-generator. The 2D-view shows the electric field $\mathbf{E}$ indicated by arrows within the ferroelectric. It is approaching $E_{c}$ in the homogeneous region.

The volume fractions of the separate domains modify during the deformation process but the basic three-domain topology remains. In the case of compressing the substrate to $\varepsilon_{i f}=0$ the simulation shows complete positive polarization in the thickness direction under the top electrode, see Fig. $10 \mathrm{c}$ ). During the second hysteresis loop the nano-generator acquires an interfacial strain up to $\varepsilon_{i f}=-0.3 \varepsilon_{0}$. A realignment of domains can be observed, depicted in Fig. 10 a) - h). This leads to a polarity reversal within the ferroelectric. The normalized hysteresis loop according to Eq. 25 for $\mathrm{P}_{z}^{a v}$ as a function of the interfacial strain $\varepsilon_{i f}$ can be seen in Fig. 11 . Correspondingly to the configurations in Fig. $10 \mathrm{a}$ ) - h) it exhibits an initial interfacial strain of $\varepsilon_{i f}=0.5 \varepsilon_{0}$ and a strain of $\varepsilon_{i f}=-0.3 \varepsilon_{0}$ under full compression.

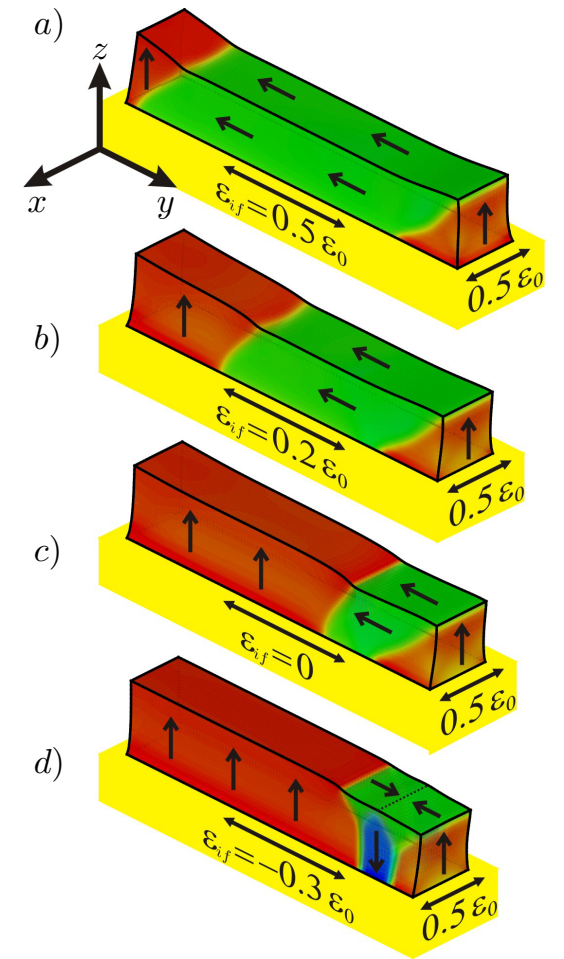

e)

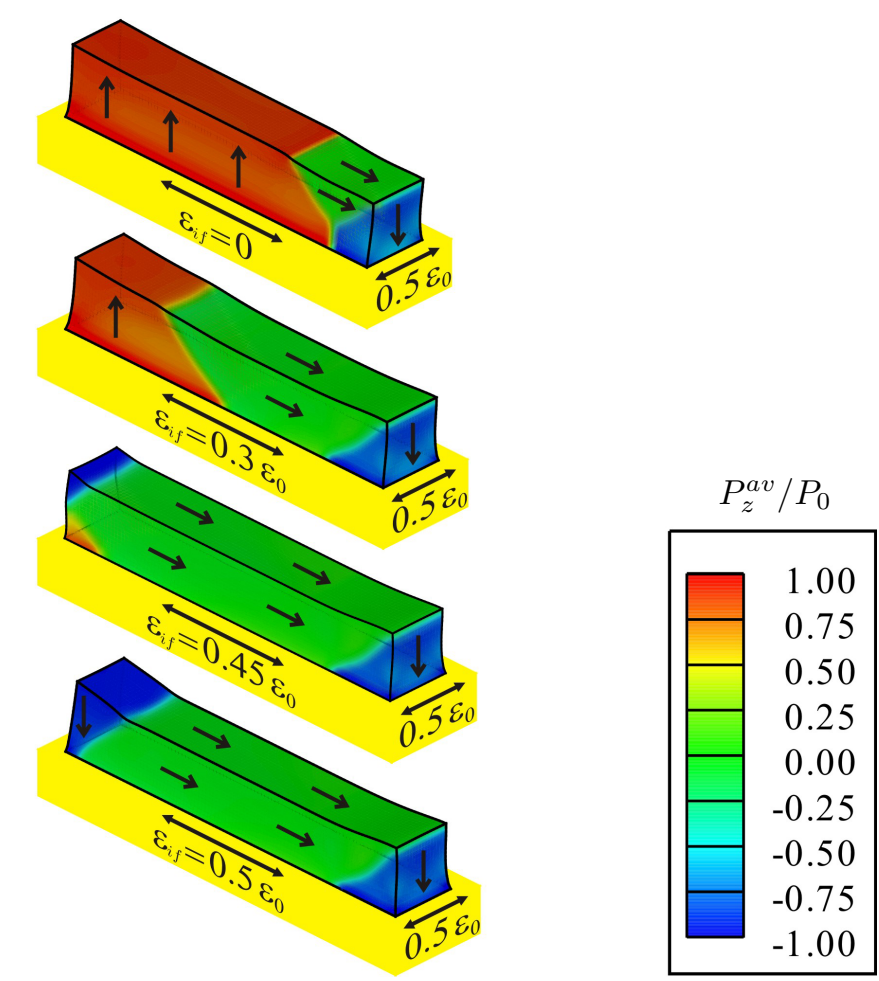

Figure 10. a) - h): Several states of domain switching under a cyclic deformation process in longitudinal direction of the ferroelectric. The electrodes are grounded. Polarization is depicted by colors and arrows and the deformation is exaggerated. 


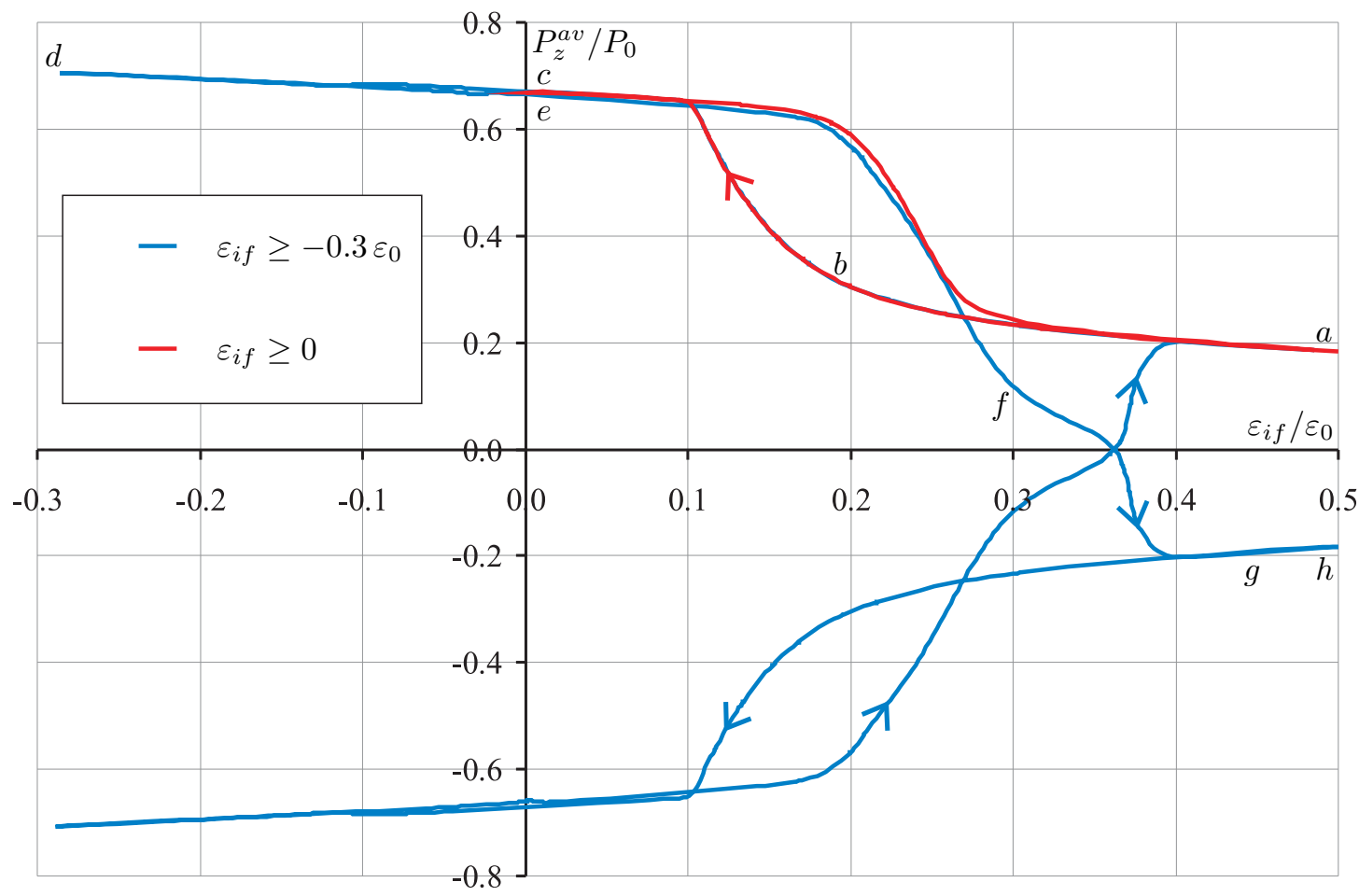

Figure 11. Resulting hysteresis graph of the polarization under a cyclic strain in the longitudinal direction. Red Line in case of strain $\varepsilon_{i f}=0.5 \varepsilon_{0}$ to $\varepsilon_{i f}=0$. Blue line polarity reversal process due to strain from $\varepsilon_{i f}=0.5 \varepsilon_{0}$ to $\varepsilon_{i f}=-0.3 \varepsilon_{0}$. States a) - h) according to Fig. 10

\section{SUMMARY AND OUTLOOK}

In this work we use a well established phase-field model for the simulation of ferroelectric material on the nanoscale. A normalization of the physical fields is presented, which has benefits for the numerical model. Using this model we discuss the influence of interfacial strain and geometrical parameters onto the materials polarization behavior. The results are in accordance to recent publications where even the transition from ferroelectric to paraelectric behavior is reported. We demonstrated that three-dimensional simulations are quite different to the solution of 2D simulations and conclude that 3D models are better suited for modeling realistic behavior. We also presented the design of a nano-generator and its performance. The design of the top electrode is essential for stabilization of interior domain mechanisms which can be utilized for electro-mechanical energy conversion.

\section{APPENDIX A. MISCELLANEOUS}

Normalized constants of the Helmholtz free energy density according to Sec. 2.1:

$$
\begin{gathered}
\sigma_{0}=\frac{E_{c} P_{0}}{\varepsilon_{0}}, \quad \bar{a}_{0}=\frac{a_{0} P_{0}}{l_{0}^{2} E_{c}}, \quad \bar{a}_{1}=\frac{a_{1} P_{0}}{E_{c}}, \quad \bar{a}_{2}=\frac{a_{2} P_{0}^{3}}{E_{c}}, \quad \bar{a}_{3}=\frac{a_{3} P_{0}^{3}}{E_{c}}, \quad \bar{a}_{4}=\frac{a_{4} P_{0}^{5}}{E_{c}}, \quad \bar{a}_{5}=\frac{a_{5} P_{0}^{7}}{E_{c}}, \quad \bar{a}_{6}=\frac{a_{6} P_{0}^{5}}{E_{c}}, \\
\bar{b}_{1}=\frac{b_{1} P_{0} \varepsilon_{0}}{E_{c}}, \quad \bar{b}_{2}=\frac{b_{2} P_{0} \varepsilon_{0}}{E_{c}}, \quad \bar{b}_{3}=\frac{b_{3} P_{0} \varepsilon_{0}}{E_{c}}, \quad \bar{c}_{1}=\frac{c_{1} \varepsilon_{0}^{2}}{E_{c} P_{0}}=\frac{c_{1} \varepsilon_{0}}{\sigma_{0}}, \quad \bar{c}_{1}=\frac{c_{2} \varepsilon_{0}^{2}}{E_{c} P_{0}}=\frac{c_{2} \varepsilon_{0}}{\sigma_{0}}, \quad \bar{c}_{1}=\frac{c_{3} \varepsilon_{0}^{2}}{E_{c} P_{0}}=\frac{c_{3} \varepsilon_{0}}{\sigma_{0}}, \\
\bar{f}_{1}=\frac{f_{1} P_{0} \varepsilon_{0}^{2}}{E_{c}}, \quad \bar{f}_{2}=\frac{f_{2} P_{0} \varepsilon_{0}^{2}}{E_{c}}, \quad \bar{f}_{3}=\frac{f_{3} P_{0} \varepsilon_{0}^{2}}{E_{c}}, \quad \bar{g}_{1}=\frac{g_{1} \varepsilon_{0} P_{0}^{3}}{E_{c}}, \quad \bar{g}_{2}=\frac{g_{2} \varepsilon_{0} P_{0}^{3}}{E_{c}}, \quad \bar{g}_{3}=\frac{g_{3} \varepsilon_{0} P_{0}^{3}}{E_{c}} .
\end{gathered}
$$


Explicit version of the normalized Helmholtz free energy equation, as suggested in Ref. ${ }^{4}$ :

$$
\begin{aligned}
& \bar{\psi}=\frac{\bar{a}_{0}}{2}\left(\bar{P}_{1,1}^{2}+\bar{P}_{2,2}^{2}+\bar{P}_{3,3}^{2}+\bar{P}_{1,2}^{2}+\bar{P}_{2,1}^{2}+\bar{P}_{1,3}^{2}+\bar{P}_{3,1}^{2}+\bar{P}_{2,3}^{2}+\bar{P}_{3,2}^{2}\right)+\frac{\bar{a}_{1}}{2}\left(\bar{P}_{1}^{2}+\bar{P}_{2}^{2}+\bar{P}_{3}^{2}\right) \\
& +\frac{\bar{a}_{2}}{4}\left(\bar{P}_{1}^{4}+\bar{P}_{2}^{4}+\bar{P}_{3}^{4}\right)+\frac{\bar{a}_{3}}{2}\left(\bar{P}_{1}^{2} \bar{P}_{2}^{2}+\bar{P}_{2}^{2} \bar{P}_{3}^{2}+\bar{P}_{1}^{2} \bar{P}_{3}^{2}\right)+\frac{\bar{a}_{4}}{6}\left(\bar{P}_{1}^{6}+\bar{P}_{2}^{6}+\bar{P}_{3}^{6}\right)+\bar{a}_{6}\left(\bar{P}_{1}^{4}\left(\bar{P}_{2}^{2}+\bar{P}_{3}^{2}\right)\right. \\
& \left.+\bar{P}_{2}^{4}\left(\bar{P}_{1}^{2}+\bar{P}_{3}^{2}\right)+\bar{P}_{3}^{4}\left(\bar{P}_{1}^{2}+\bar{P}_{2}^{2}\right)\right)+\frac{\bar{a}_{5}}{4}\left(\bar{P}_{1}^{4} \bar{P}_{2}^{4}+\bar{P}_{2}^{4} \bar{P}_{3}^{4}+\bar{P}_{1}^{4} \bar{P}_{3}^{4}\right)-\frac{\bar{b}_{1}}{2}\left(\bar{\varepsilon}_{11} \bar{P}_{1}^{2}+\bar{\varepsilon}_{22} \bar{P}_{2}^{2}+\bar{\varepsilon}_{33} \bar{P}_{3}^{2}\right) \\
& -\frac{\bar{b}_{2}}{2}\left(\left(\bar{\varepsilon}_{22}+\bar{\varepsilon}_{33}\right) \bar{P}_{1}^{2}+\left(\bar{\varepsilon}_{11}+\bar{\varepsilon}_{33}\right) \bar{P}_{2}^{2}+\left(\bar{\varepsilon}_{11}+\bar{\varepsilon}_{22}\right) \bar{P}_{3}^{2}\right)-\bar{b}_{3}\left(\left(\bar{\varepsilon}_{12}+\bar{\varepsilon}_{21}\right) \bar{P}_{1} \bar{P}_{2}+\left(\bar{\varepsilon}_{13}+\bar{\varepsilon}_{31}\right) \bar{P}_{1} \bar{P}_{3}\right. \\
& \left.+\left(\bar{\varepsilon}_{23}+\bar{\varepsilon}_{32}\right) \bar{P}_{2} \bar{P}_{3}\right)+\frac{\bar{c}_{1}}{2}\left(\bar{\varepsilon}_{11}^{2}+\bar{\varepsilon}_{22}^{2}+\bar{\varepsilon}_{33}^{2}\right)+\bar{c}_{2}\left(\bar{\varepsilon}_{11} \bar{\varepsilon}_{22}+\bar{\varepsilon}_{11} \bar{\varepsilon}_{33}+\bar{\varepsilon}_{22} \bar{\varepsilon}_{33}\right) \\
& +\frac{\bar{c}_{3}}{2}\left(\bar{\varepsilon}_{12}^{2}+\bar{\varepsilon}_{21}^{2}+\bar{\varepsilon}_{13}^{2}+\bar{\varepsilon}_{31}^{2}+\bar{\varepsilon}_{23}^{2}+\bar{\varepsilon}_{32}^{2}\right)+\left(\frac{f_{1}}{2} \bar{\varepsilon}_{11}^{2}+\frac{f_{2}}{2}\left(\bar{\varepsilon}_{22}^{2}+\bar{\varepsilon}_{33}^{2}\right)+f_{3}\left(\bar{\varepsilon}_{11} \bar{\varepsilon}_{22}+\bar{\varepsilon}_{11} \bar{\varepsilon}_{33}\right)\right. \\
& \left.+f_{4} \bar{\varepsilon}_{22} \bar{\varepsilon}_{33}+\frac{f_{5}}{2}\left(\bar{\varepsilon}_{12}^{2}+\bar{\varepsilon}_{21}^{2}+\bar{\varepsilon}_{13}^{2}+\bar{\varepsilon}_{31}^{2}\right)+\frac{f_{6}}{2}\left(\bar{\varepsilon}_{23}^{2}+\bar{\varepsilon}_{32}^{2}\right)\right) \bar{P}_{1}^{2}+\left(\frac{f_{1}}{2} \bar{\varepsilon}_{22}^{2}+\frac{f_{2}}{2}\left(\bar{\varepsilon}_{11}^{2}+\bar{\varepsilon}_{33}^{2}\right)\right. \\
& \left.+f_{3}\left(\bar{\varepsilon}_{11} \bar{\varepsilon}_{22}+\bar{\varepsilon}_{22} \bar{\varepsilon}_{33}\right)+f_{4} \bar{\varepsilon}_{11} \bar{\varepsilon}_{33}+\frac{f_{5}}{2}\left(\bar{\varepsilon}_{12}^{2}+\bar{\varepsilon}_{21}^{2}+\bar{\varepsilon}_{23}^{2}+\bar{\varepsilon}_{32}^{2}\right)+\frac{f_{6}}{2}\left(\bar{\varepsilon}_{13}^{2}+\bar{\varepsilon}_{31}^{2}\right)\right) \bar{P}_{2}^{2} \\
& +\left(\frac{f_{1}}{2} \bar{\varepsilon}_{33}^{2}+\frac{f_{2}}{2}\left(\bar{\varepsilon}_{11}^{2}+\bar{\varepsilon}_{22}^{2}\right)+f_{3}\left(\bar{\varepsilon}_{11} \bar{\varepsilon}_{33}+\bar{\varepsilon}_{22} \bar{\varepsilon}_{33}\right)+f_{4} \bar{\varepsilon}_{11} \bar{\varepsilon}_{22}+\frac{f_{5}}{2}\left(\bar{\varepsilon}_{13}^{2}+\bar{\varepsilon}_{31}^{2}+\bar{\varepsilon}_{23}^{2}+\bar{\varepsilon}_{32}^{2}\right)\right. \\
& \left.+\frac{f_{6}}{2}\left(\bar{\varepsilon}_{12}^{2}+\bar{\varepsilon}_{21}^{2}\right)\right) \bar{P}_{3}^{2}+\left(\frac{g_{1}}{4} \bar{\varepsilon}_{11}+\frac{g_{2}}{4}\left(\bar{\varepsilon}_{22}+\bar{\varepsilon}_{33}\right)\right) \bar{P}_{1}^{4}+\left(\frac{g_{1}}{4} \bar{\varepsilon}_{22}+\frac{g_{2}}{4}\left(\bar{\varepsilon}_{11}+\bar{\varepsilon}_{33}\right)\right) \bar{P}_{2}^{4} \\
& +\left(\frac{g_{1}}{4} \bar{\varepsilon}_{33}+\frac{g_{2}}{4}\left(\bar{\varepsilon}_{11}+\bar{\varepsilon}_{22}\right)\right) \bar{P}_{3}^{4}+\frac{g_{3}}{4}\left(\bar{\varepsilon}_{12}+\bar{\varepsilon}_{21}\right)\left(\bar{P}_{1} \bar{P}_{2}^{3}+\bar{P}_{2} \bar{P}_{1}^{3}\right)+\frac{g_{3}}{4}\left(\bar{\varepsilon}_{13}+\bar{\varepsilon}_{31}\right)\left(\bar{P}_{1} \bar{P}_{3}^{3}+\bar{P}_{3} \bar{P}_{1}^{3}\right) \\
& +\frac{g_{3}}{4}\left(\bar{\varepsilon}_{23}+\bar{\varepsilon}_{32}\right)\left(\bar{P}_{2} \bar{P}_{3}^{3}+\bar{P}_{3} \bar{P}_{2}^{3}\right)+\frac{1}{2 \kappa_{0}}\left(\left(\bar{D}_{1}-\bar{P}_{1}\right)^{2}+\left(\bar{D}_{2}-\bar{P}_{2}\right)^{2}+\left(\bar{D}_{3}-\bar{P}_{3}\right)^{2}\right)
\end{aligned}
$$

With the normalized constants

$$
\begin{array}{ccccccc}
\bar{a}_{0}=1 & \bar{a}_{1}=-0.668325 & \bar{a}_{2}=-3.80563 & \bar{a}_{3}=0.78922 & \bar{a}_{4}=12.4421 & \bar{a}_{5}=368 & \bar{a}_{6}=0.134226 \\
\bar{b}_{1}=2.54138 & \bar{b}_{2}=1.74267 & \bar{b}_{3}=0.399353 & \bar{c}_{1}=2.04999 & \bar{c}_{2}=0.971673 & \bar{c}_{3}=2.55952 \\
\bar{f}_{1}=0.663581 & \bar{f}_{2}=0.841326 & \bar{f}_{3}=-0.170635 & \bar{f}_{4}=0.687281 & \bar{f}_{5}=0.21328 & \bar{f}_{6}=0.426588 \\
\multicolumn{8}{c}{\bar{g}_{1}=-3.66149} & \bar{g}_{2}=6.27423 & \bar{g}_{3}=-1.21644
\end{array}
$$

\section{REFERENCES}

[1] E. Fried, M. E. Gurtin: Continuum theory of thermally induced phase transitions based on an order parameter, Physica D 68, 326-343, 1993.

[2] E. Fried, M. E. Gurtin: Dynamic solid-solid transitions with phase characterized by an order parameter, Physica D 72, 287-308, 1994.

[3] M. E. Gurtin: Generalized Ginzburg-Landau and Cahn-Hilliard equations based on a microforce balance, Physica D 92, 178-192, 1996.

[4] A. Kontsos, C. M. Landis: Computational modeling of domain wall interactions with dislocations in ferroelectric crystals. International Journal of Solids and Structures 46: 1491-1498, 2009.

[5] Y. Su, C. M. Landis: Continuum thermodynamics of ferroelectric domain evolution: Theory, finite element implementation, and application to domain wall pinning. Journal of the Mechanics and Physics of Solids 55, 280-305, 2007.

[6] J. X. Zhang, R.Wu, S. Choudhury, Y. L. Li, S. Y. Hu and L. Q. Chen: Three-dimensional phase-field simulation of domain structures in ferroelectric islands. Applied Physics Letters 92, Art. No. 122906J, 2008.

[7] J. F. Scott: Applications of Modern Ferroelectrics. Science 315: 954-959, 2007. 
[8] M. E. Lines, A. M. Glass: Principles and Applications of Ferroelectrics and Related Materials. Clarendon, Oxford, 1977.

[9] Y. Xu: Ferroelectric Materials and their Applications. Elsevier, Amsterdam, 1991.

[10] D. D. Fong, G. B. Stephenson, S. K. Streiffer, J. A. Eastman, O. Auciello, P. H. Fuoss, C. Thompson: Ferroelectricity in Ultrathin Perovskite Films. Science 304: 1650-1653, 2004.

[11] J. Junquera, P. Ghosez: Critical thickness for ferroelectricity in perovskite ultrathin films. Letters to nature 422: 506-509, DOI 10.1038/nature01501, 2003.

[12] L. Qiao, X. Bi: Origin of compressive strain and phase transition characteristics of thin $\mathrm{BaTiO}_{3}$ film grown on $\mathrm{LaNiO}_{3} / \mathrm{Si}$ substrate. Phys. Status Solidi 11: 2511-1516, DOI 10.1002/pssa.201026271, 2010.

[13] S. Rios, J. F. Scott, A. Lookman, J. McAneney, R. M. Bowman, J. M. Gregg: Phase transitions in epitaxial Ba0.5Sr0.5TiO3 thin films. J. Appl. Physics 99: 024107, 2006.

[14] K. J. Choi, M. Biegalski, Y. L. Li, A. Sharan, J. Schubert, R. Uecker, P. Reiche, Y. B. Chen, X. Q. Pan, V. Gopalan, L.-Q. Chen, D. G. Schlom, C. B. Eom: Enhancement of Ferroelectricity in Strained BaTiO3 Thin Films. Science 306: 1005-1009, 2004.

[15] H. Li, A. L. Roytburd, S. P. Alpay, T. D. Tran, L. Salamanca-Riba, R. Ramesh: Dependence of dielectric properties on internal stresses in epitaxial barium strontium titanate thin films. Applied Physics Letters 78(16), 2001.

[16] A. Kontsos, C. M. Landis: Phase-Field Modeling of Domain Structure Energetics and Evolution in Ferroelectric Thin Films. Journal of Applied Mechanics 77: 041014, 2010.

[17] Q. Y. Qiu, V. Nagarajan, S. P. Alpay: Film thickness versus misfit strain phase diagrams for epitaxial PbTiO3 ultrathin ferroelectric films. Physical Review B 78: 064117, 2008.

[18] Y. L. Li, L. Q. Chen: Temperature-strain phase diagram for BaTiO3 thin films. Applied Physics Letters 88: $072905,2006$.

[19] C. Ederer, N. A. Spaldin: Effect of Epitaxial Strain on the Spontaneous Polarization of Thin Film Ferroelectrics. Physical Review Letters 95, 2005.

[20] Z.-G. Ban and S. P. Alpay: Optimization of the tenability of barium strontium titanate films via epitaxial stresses. Appl. Phys. Lett. 93(1): 504, 2003.

[21] A. L. Roytburd, S. P. Alpay, V. Nagarajan, C. S. Ganpule, S. Aggarwal, E. D. Williams, R. Ramesh: Measurement of Internal Stresses via the Polarization in Epitaxial Ferroelectric Films. Physical Review Letters $85(1), 2000$.

[22] D. G. Schlom, L.-Q. Chen, C.-B. Eom, K. M. Rabe, S. K. Streiffer, J.-M. Triscone: Strain Tuning of Ferroelectric Thin Films. Annu. Rev. Mater. Res. 37: 589-626, 2007.

[23] L.-Q. Chen: Phase-Field Method of Phase Transitions/Domain Structures in Ferroelectric Thin Films: A Review. J. Am. Ceram. Soc. 91(6) DOI: 10.1111/j.1551-2916.2008.02413.x, 2008.

[24] S. Choudhury, Y. Li, L.-Q. Chen: A Phase Diagram for Epitaxial $\mathrm{PbZr}_{1-x} \operatorname{Ti}_{x} \mathrm{O}_{3}$ Thin Films at the Bulk Morphotropic Boundary Composition. J. Am. Ceram. Soc. 88(6) DOI: 10.1111/j.1551-2916.2005.00319.x, 2005.

[25] K. Dayal, K. Bhattacharya: A real-space non-local phase-field model of ferroelectric domain patterns in complex geometries. Acta Materialia 55: 1907-1917, 2007.

[26] B.-X. Xu, D. Schrade, R. Mller, D. Gross, T. Granzow, J. Rdel: Phase field simulation and experimental investigation of the electro-mechanical behavior of ferroelectrics. Zeitschrift fr Angewandte Mathematik und Mechanik 90(7) DOI: 10.1002/zamm.200900344, 2010.

[27] A. Devonshire: Theory of Ferroelectrics. Phils. Mag. 3(10), 85-130, 1954.

[28] J. Hlinka, P. Mrton: Phenomenological model of a 90 domain wall in $\mathrm{BaTiO}_{3}$-type ferroelectrics. Physical Review B 74, 1098-0121/2006/74(10)/104104(12), 2006. 